International Mathematical Forum, 1, 2006, no. 36, 1757-1762

\title{
Sufficient conditions of analytic functions involving the generalized fractional integral operator
}

\author{
B. A. Frasin \\ Department of Mathematics \\ $\mathrm{Al}$ al-Bayt University \\ P.O. Box:130095 \\ Mafraq, Jordan \\ bafrasin@yahoo.com.
}

\begin{abstract}
In this paper, we determine the sufficient conditions that make the generalized fractional integral operator $J_{0, z}^{\beta, \gamma, \delta}$ to be in the comprehensive family $\mathcal{E}(\Phi, \Psi ; \alpha)$ of analytic functions of order $\alpha$ which contains various well-known classes of analytic functions as well as many new ones.
\end{abstract}

Mathematics Subject Classification: 30C45

Keywords: Analytic functions, Starlike and Convex functions.

\section{Introduction}

Let $\mathcal{A}$ denote the class of functions of the form

$$
f(z)=z+\sum_{n=2}^{\infty} a_{n} z^{n}
$$

which are analytic in the open unit $\operatorname{disk} \mathcal{U}=\{z:|z|<1\}$. Denote by $\mathcal{S}^{*}(\alpha)$ the class of starlike functions $f \in \mathcal{A}$ of order $\alpha(0 \leq \alpha<1)$ satisfying

$$
\operatorname{Re}\left(\frac{z f^{\prime}(z)}{f(z)}\right)>\alpha \quad(z \in \mathcal{U})
$$

and let $\mathcal{C}(\alpha)$ be the class of convex functions $f \in \mathcal{A}$ of order $\alpha(0 \leq \alpha<1)$ such that $z f^{\prime}(z) \in \mathcal{S}^{*}(\alpha)$. Further, let $\mathcal{P}(\alpha)$ denote the class of functions $f \in \mathcal{A}$ such that

$$
\operatorname{Re}\left(f^{\prime}(z)\right)>\alpha \quad(0 \leq \alpha<1, z \in \mathcal{U})
$$


Given two functions $f, h \in \mathcal{A}, f(z)=z+\sum_{n=2}^{\infty} a_{n} z^{n}$ and $h(z)=z+\sum_{n=2}^{\infty} c_{n} z^{n}$ their convolution or Hadamard product $f(z) * h(z)$, is defined by

$$
f(z) * h(z)=z+\sum_{n=2}^{\infty} a_{n} c_{n} z^{n} \quad(z \in \mathcal{U})
$$

Ruscheweyh [3] using the convolution techniques, introduced and studied an important subclass of $\mathcal{A}$, the class of prestarlike functions of order $\alpha$, which is denoted by $\mathcal{R}(\alpha)$. Thus $f \in \mathcal{A}$ is said to be prestarlike function of order $\alpha(0 \leq \alpha<1)$ if $f * s_{\alpha} \in \mathcal{S}^{*}(\alpha)$ where $s_{\alpha}(z)=z(1-z)^{2 \alpha-2}=$ $z+\sum_{n=2}^{\infty} c(\alpha, n) z^{n} ; c(\alpha, n)=\prod_{k=2}^{n} \frac{k-2 \alpha}{(n-1) !} \quad(n \geq 2)$. It may be noted that $\mathcal{R}(0)=$ $\mathcal{C}(0)$ and $\mathcal{R}(1 / 2)=\mathcal{S}^{*}(1 / 2)$.

Juneja et al. [1] define the family $\mathcal{E}(\Phi, \Psi ; \alpha)$ consisting of the functions $f \in \mathcal{A}$ so that

$$
\operatorname{Re}\left(\frac{f(z) * \Phi(z)}{f(z) * \Psi(z)}\right)>\alpha \quad(z \in \mathcal{U})
$$

where $\alpha(0 \leq \alpha<1), \Phi(z)=z+\sum_{n=2}^{\infty} \lambda_{n} z^{n}$ and $\Psi(z)=z+\sum_{n=2}^{\infty} \mu_{n} z^{n}$ are analytic in $\mathcal{U}$ with the conditions $\lambda_{n} \geq 0, \mu_{n} \geq 0, \lambda_{n} \geq \mu_{n}$ for $n \geq 2$, and $f(z) * \Psi(z) \neq 0$.

It is easy to check that various subclasses of $\mathcal{A}$ referred to above can be represented as $\mathcal{E}(\Phi, \Psi ; \alpha)$ for suitable choices of $\Phi, \Psi$. For example

(i) $\mathcal{E}\left(\frac{z}{(1-z)^{2}}, \frac{z}{1-z} ; \alpha\right)=\mathcal{S}^{*}(\alpha)$;

(ii) $\mathcal{E}\left(\frac{z+z^{2}}{(1-z)^{3}}, \frac{z}{(1-z)^{2}} ; \alpha\right)=\mathcal{C}(\alpha)$

(iii) $\mathcal{E}\left(\frac{z}{(1-z)^{2}}, z ; \alpha\right)=\mathcal{P}(\alpha)$

(iv) $\mathcal{E}\left(\frac{z+(1-2 \alpha) z^{2}}{(1-z)^{3-2 \alpha}}, \frac{z}{(1-z)^{2-2 \alpha}} ; \alpha\right)=\mathcal{R}(\alpha)$.

In fact many new subclasses of $\mathcal{A}$ can be defined and studied by suitably choosing $\Phi(z)$ and $\Psi(z)$. Thus

$(v) \mathcal{E}\left(\frac{z+z^{2}}{(1-z)^{3}}, z ; \alpha\right)=\left\{f \in \mathcal{A}: \operatorname{Re}\left(\left(z f^{\prime}(z)\right)^{\prime}\right)>\alpha\right\}$

(vi) $\mathcal{E}\left((1-\delta) \frac{z}{(1-z)^{2}}+\delta \frac{z+z^{2}}{(1-z)^{3}}, z ; \alpha\right)$

$$
=\left\{f \in \mathcal{A}: \operatorname{Re}\left((1-\delta) f^{\prime}(z)+\delta\left(z f^{\prime}(z)\right)^{\prime}\right)>\alpha\right\} \text { and so on. }
$$


Let $F(a, b ; c ; z)$ be the Gauss hypergeometric function defined, for $z \in \mathcal{U}$, by

$$
F(a, b ; c ; z)=\sum_{n=0}^{\infty} \frac{(a)_{n}(b)_{n}}{(c)_{n}(1)_{n}}
$$

where $(\nu)_{n}$ is the Pochhammer symbol defined by

$$
(\nu)_{n}=\frac{\Gamma(\nu+n)}{\Gamma(\nu)}= \begin{cases}1 & (n=0) \\ \nu(\nu+1)(\nu+2) \cdots(\nu+n-1) & (n \in \mathbb{N})\end{cases}
$$

We need the following definitions of fractional integral operator given by Srivastava et al. [2].

Definition 1.For real number $\lambda>0, \gamma$ and $\delta$, the fractional integral operator $I_{0, z}^{\lambda, \gamma, \delta}$ is defined by

$$
I_{0, z}^{\beta, \gamma, \delta} f(z)=\frac{z^{-\beta-\gamma}}{\Gamma(\beta)} \int_{0}^{z}(z-t)^{\lambda-1} F(\beta+\gamma,-\delta ; \beta ; 1-t / z) f(t) d t
$$

where a function $f(z)$ is analytic in a simply-connected region of the $z$-plane containing the origin with the order

$$
f(z)=O\left(|z|^{\varepsilon}\right) \quad(z \longrightarrow 0)
$$

with $\varepsilon>\max \{0, \gamma-\delta\}-1$, and the multiplicity of $(z-t)^{\lambda-1}$ is removed by requiring $\log (z-t)$ to be real when $z-t>0$.

Definition 2.Under the hypotheses of Definition 1, let

$$
\beta>0, \quad \min \{\beta+\delta,-\gamma+\delta,-\gamma\}>-2, \text { and } 3 \beta \geq \gamma(\beta+\eta)
$$

Then the fractional integral operator $J_{0, z}^{\beta, \gamma, \delta}$ is defined by

$$
J_{0, z}^{\beta, \gamma, \delta} f(z)=\frac{\Gamma(2-\gamma) \Gamma(2+\beta+\eta)}{\Gamma(2-\gamma+\eta)} z^{\gamma} I_{0, z}^{\beta, \gamma, \delta} f(z)
$$

\section{Main Results}

In order to derive our results, we need the following lemmas.

Lemma 1([2]). If $\beta>0$ and $n>\gamma-\delta-1$, then 


$$
I_{0, z}^{\beta, \gamma, \delta} z^{n}=\frac{\Gamma(n+1) \Gamma(n-\gamma+\delta+1)}{\Gamma(n-\gamma+1) \Gamma(n+\beta+\delta+1)} z^{n-\gamma} .
$$

Lemma $2([\mathbf{1}])$. Let the functions $f(z)$ defined by (1) satisfies

$$
\sum_{n=2}^{\infty} \frac{\lambda_{n}-\alpha \mu_{n}}{1-\alpha} a_{n} \leq 1
$$

then $f(z) \in \mathcal{E}(\Phi, \Psi ; \alpha)$. The result (12) is sharp.

Now we prove

Theorem 1. Under the constraints (9), if the function $f(z)$ defined by (1) satisfies

$$
\sum_{n=2}^{\infty} \frac{\lambda_{n}-\alpha \mu_{n}}{1-\alpha} a_{n} \leq \frac{(2-\gamma)(2+\beta+\delta)}{2(2-\gamma+\delta)}
$$

then $J_{0, z}^{\beta, \gamma, \delta} f(z) \in \mathcal{E}(\Phi, \Psi ; \alpha)$.

Proof. With the aid of Lemma 1 and Definition 2, we have

$$
J_{0, z}^{\beta, \gamma, \delta} f(z)=z+\sum_{n=2}^{\infty} \Delta(n) a_{n} z^{n}
$$

where

$$
\Delta(n)=\frac{(2-\gamma+\delta)_{n-1}(1)_{n}}{(2-\gamma)_{n-1}(2+\beta+\delta)_{n-1}} \quad(n \geq 2)
$$

we can see that the function $\Delta(n)$ is non-increasing for integers $n \geq 2$, and we have

$$
0<\Delta(n) \leq \Delta(2)=\frac{2(2-\gamma+\delta)}{(2-\gamma)(2+\beta+\delta)} .
$$

Therefore, by using (13) and (15), we have

$$
\sum_{n=2}^{\infty} \frac{\lambda_{n}-\alpha \mu_{n}}{1-\alpha} \Delta(n) a_{n} \leq \Delta(2) \sum_{n=2}^{\infty} \frac{\lambda_{n}-\alpha \mu_{n}}{1-\alpha} \leq 1 .
$$

Hence, by Lemma $2, J_{0, z}^{\beta, \gamma, \delta} f(z) \in \mathcal{E}(\Phi, \Psi ; \alpha)$.

Putting $\Phi(z)=z /(1-z)^{2}$ and $\Psi(z)=z /(1-z)$ in Theorem 1 , we have Corollary 1. Under the constraints (9), if the function $f(z)$ defined by (1) satisfies 


$$
\sum_{n=2}^{\infty}(n-\alpha) a_{n} \leq \frac{(1-\alpha)(2-\gamma)(2+\beta+\delta)}{2(2-\gamma+\delta)},
$$

then $J_{0, z}^{\beta, \gamma, \delta} f(z) \in \mathcal{S}^{*}(\alpha)$.

Putting $\Phi(z)=\left(z+z^{2}\right) /(1-z)^{3}$ and $\Psi(z)=z /(1-z)^{2} 1 / 2$ in Theorem 1 , we have

Corollary 2. Under the constraints (9), if the function $f(z)$ defined by (1) satisfies

$$
\sum_{n=2}^{\infty} n(n-\alpha) a_{n} \leq \frac{(1-\alpha)(2-\gamma)(2+\beta+\delta)}{2(2-\gamma+\delta)},
$$

then $J_{0, z}^{\beta, \gamma, \delta} f(z) \in \mathcal{C}(\alpha)$.

Putting $\Phi(z)=z /(1-z)^{2}$ and $\Psi(z)=z$ in Theorem 1, we have

Corollary 3. Under the constraints (9), if the function $f(z)$ defined by (1) satisfies

$$
\sum_{n=2}^{\infty} n a_{n} \leq \frac{(1-\alpha)(2-\gamma)(2+\beta+\delta)}{2(2-\gamma+\delta)}
$$

then $J_{0, z}^{\beta, \gamma, \delta} f(z) \in \mathcal{P}(\alpha)$.

Putting $\Phi(z)=\left(z+(1-2 \beta) z^{2}\right) /(1-z)^{3-2 \alpha}$ and $\Psi(z)=z /(1-z)^{2-2 \alpha}$ in Theorem 1, we have

Corollary 4. Under the constraints (9), if the function $f(z)$ defined by (1) satisfies

$$
\sum_{n=2}^{\infty} C(\alpha, n)(n-\alpha) a_{n} \leq \frac{(1-\alpha)(2-\gamma)(2+\beta+\delta)}{2(2-\gamma+\delta)}
$$

then $J_{0, z}^{\beta, \gamma, \delta} f(z) \in \mathcal{R}(\alpha)$.

Next we prove

Theorem 2. Let

$$
h(z)=z+\sum_{n=2}^{\infty} \frac{(2-\gamma+\delta)_{n-1}(1)_{n}}{(2-\gamma)_{n-1}(2+\beta+\delta)_{n-1}} z^{n}
$$

be in the class $\mathcal{E}\left(z f^{\prime}(z), f(z) ; \alpha\right)$, then $J_{0, z}^{\beta, \gamma, \delta} f(z) \in \mathcal{S}^{*}(\alpha)$.

Proof. Notice from (14) and (21) that 


$$
J_{0, z}^{\beta, \gamma, \delta} f(z)=z+\sum_{n=2}^{\infty} \frac{(2-\gamma+\delta)_{n-1}(1)_{n}}{(2-\gamma)_{n-1}(2+\beta+\delta)_{n-1}} a_{n} z^{n}=(h * f)(z),
$$

which readily yields

$$
\frac{z\left(J_{0, z}^{\beta, \gamma, \delta} f(z)\right)^{\prime}}{J_{0, z}^{\beta, \gamma, \delta} f(z)}=\frac{z(h * f)^{\prime}(z)}{(h * f)(z)}=\frac{\left(h *\left(z f^{\prime}\right)(z)\right.}{(h * f)(z)} .
$$

Setting $\Phi(z)=z(f(z)$ and $\Psi(z)=f(z)$ in (23), we find that

$$
\operatorname{Re}\left(\frac{z\left(J_{0, z}^{\beta, \gamma, \delta} f(z)\right)^{\prime}}{J_{0, z}^{\beta, \gamma, \delta} f(z)}\right)>\alpha
$$

which implies that $J_{0, z}^{\beta, \gamma, \delta} f(z) \in \mathcal{S}^{*}(\alpha)$.

\section{References}

[1] O.P. Juneja, T.R. Reddy and M.L. Mogra, A convolution approach for analytic functions with negative coefficients, Soochow J. Math. 11 (1985), 69-81.

[2] H.M. Srivastava, M. Saigo and S. Owa, A class of distortion theorems involving certain operators of fractional calculus, J. Math. Anal. Appl. 131 (1988), 412-420.

[3] S. Ruscheweyh, Linear operators between classes of prestarlike functions, Comment. Math. Helv.52 (1977), 497- 509.

Received: October 9, 2005 